\title{
DC Power System for Superconducting Coils of Fusion Plasma Test Facility LHD
}

\author{
Hirotaka Chikaraishi Member (NIFS) \\ Sigeyuki Takami Non-member (NIFS) \\ Tomoyuki Inoue Non-member (NIFS) \\ Satoru Sakakibara Non-member (NIFS) \\ Toshifumi Ise Member (Osaka University) \\ Tomoyuki Haga Non-member (Shinko Eng. Co., Ltd.) \\ Hiroyuki Niwa Member (Aichi Electric Co., Ltd.)
}

Keywords: magnetic coupling, current control, dc power supply, LHD, H infinity control

The Large Helical Device (LHD) operating at the National Institute for Fusion Science (NIFS) is a fusion experimental facility based on a set of superconducting coils. The size of the LHD is about $16 \mathrm{~m}$ in diameter and $8 \mathrm{~m}$ in height, and it is also the largest superconducting machine in the world under operation. This machine has twelve superconducting coils to form the magnetic field to confine plasma and six power supplies to magnetize the coils. The output voltages of power supplies are only $33 \mathrm{~V}$ or $45 \mathrm{~V}$ in spite of the large operating current of from $16 \mathrm{kA}$ to $24 \mathrm{kA}$. So we use a 12-phase double star connected thyristor rectifier for each power supply. For the power supplies, following terms are required to this power system.

- Current measurement error is less than $(0.03 \%$ of reading value

$$
+4.5 \mathrm{~A} \text { ) }
$$

- Steady state current control error is less than $(0.04 \%$ of set value $+6 \mathrm{~A}$ )

- Current overshoot at normal ramp up rate is not allowed

- Settling time for $0.1 \%$ is less than $1 \mathrm{~s}$ for normal ramp-up rate

In the design of a control system, the electrical parameters of the superconducting coil system are required to determine the control gains. The inductance matrix of superconducting coils and structures was measured from the coil terminals, Also the mutual inductance between plasma and coils are important and we estimate them using coil current deviations induced by plasma current.

For the LHD power supplies, we designed and tested five control schemes as following.

$P I$ or $P$ control They are simple but stable controller. The time constants of all current responses are same and the current balance is kept constant while currents are changing. But the control gain is limited by stability condition, the better response is difficult to built.

$H \infty$ control

advanced $H \infty$ control (1) This controller has a feed forward using plasma current signal and keep the coil currents constant even if the plasma current flows in the device.

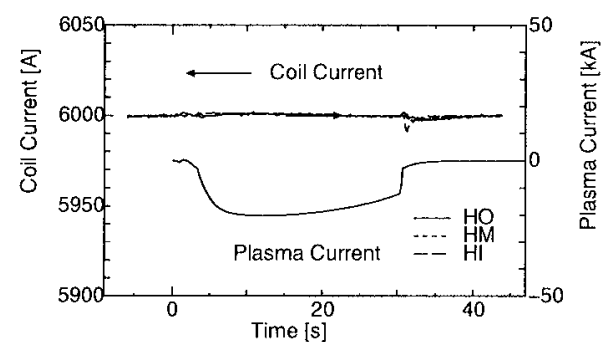

Fig. 1. Reaction caused by plasma current. $H \infty(1)$ control is applied

advanced $H \infty$ control (2) This control keep interlinked magnetic flux constant and allows the coil current deviation when plasma current flows in the LHD.

advanced $P$ controller This controller includes current error observer to cancel the steady state error and the feed forward of $L d i_{c} / d t$ to make current response better.

The operator of LHD power system can select one of these control schemes even if the power supplies are exciting the coils. With this ability, the power system becomes more useful for plasma experiments.

Figure 1 shows an example of operation using the $H \infty(1)$. This current controler keeps current constant while the plasma burning. The coil currents and terminal voltages are not disturbed so large when plasma discharge is finished.

\section{References}

( 1 ) A. Iiyoshi et al.: Nuclear Fusion, Vol.39, spec. issue., pp.1245-1256 (1999)

( 2 ) O. Motojima et al.: Fusion Eng. and Design, Vol.20, pp.3-14 (1993-1)

( 3 ) T. Satow: Cryogenics, Vol.38, pp.1145-1153 (1998-11)

(4) S. Tanahashi et al.: Fusion Eng. and Design, Vol.20, pp.107-111 (1993-1)

( 5 ) T. Ise et al.: PESC 96 Record. 27th Annual IEEE Power Electronics Specialists Conf., pp.1099-1104 (1996)

( 6 ) H. Chikaraishi et al.: IEEE Trans. on Applied Super., Vol.12, No.1, pp.13741377 (2002) 


\section{Power System for Superconducting Coils of Fusion Plasma Test Facility LHD}

$\begin{array}{ll}\text { Hirotaka Chikaraishi* } & \text { Member } \\ \text { Sigeyuki Takami* }^{*} & \text { Non-member } \\ \text { Tomoyuki Inoue* }^{*} & \text { Non-member } \\ \text { Satoru Sakakibara }^{*} & \text { Non-member } \\ \text { Toshifumi Ise } & \text { Member } \\ \text { Tomoyuki Haga a** }^{* * *} & \text { Non-member } \\ \text { Hiroyuki Niwa }^{* * * *} & \text { Member }\end{array}$

The Large Helical Device (LHD) is an experimental facility for fusion plasma using a large scale mutually coupled superconducting coil system and it is now operating at the National Institute for Fusion Science. This paper introduces the design and the operation results of the dc power systems to drive these coils. First, the outline of the LHD and the power supplies are described. Next, a control system and the current controller for the power supplies are introduced. Finally, some experimental results are introduced.

Keywords: magnetic coupling, current control, dc power supply, LHD

\section{Introduction}

The Large Helical Device (LHD) that is operating at the National Institute for Fusion Science (NIFS) is a fusion experimental facility using superconducting coils. The size of the LHD is about $16 \mathrm{~m}$ in diameter and $8 \mathrm{~m}$ in height, and it is also the largest superconducting device in the world under operation. This device has twelve superconducting coils to form the magnetic field to confine plasma ${ }^{(1)(2)}$. The strength of the magnetic field is $3 \mathrm{~T}$ at the plasma center and amount of stored energy reaches to $1 \mathrm{GJ}$.

In the following sections, first we describe the outline of LHD and its operation scenario. Next, we present the design of the power supplies, which consist of thyristor rectifiers, dc filters and a computer control system. After that, the current control schemes are described and some operation results for each control scheme are introduced.

\section{Outline of the LHD and its Operation}

Figure 1 shows a cross section of LHD that includes superconducting coils. The LHD has six poloidal coils designated as OV-L, OV-U, IS-L, IS-U, IV-L, and IV-U, and two helical coil sets designated as $\mathrm{H} 1$ and $\mathrm{H} 2$, as displayed in the figure $^{(3)}$. Each of the helical coils is divided in three sections

\footnotetext{
* National Institute for Fusion Science

hchikara@nifs.ac.jp

Oroshi, 322-6, Tokishi, Gifu, 509-5292, Japan

** Osaka University

Suitashi, Osaka, 565-0871, Japan

*** Shinko Engineering \& Maintenance Co., Ltd. Iwayakita, Nadaku, Kobeshi, Hyougo, Japan

**** Aichi Electric Co., Ltd.

1, Aichicho, Kasugaishi, Aichi. 486-8666, Japan
}

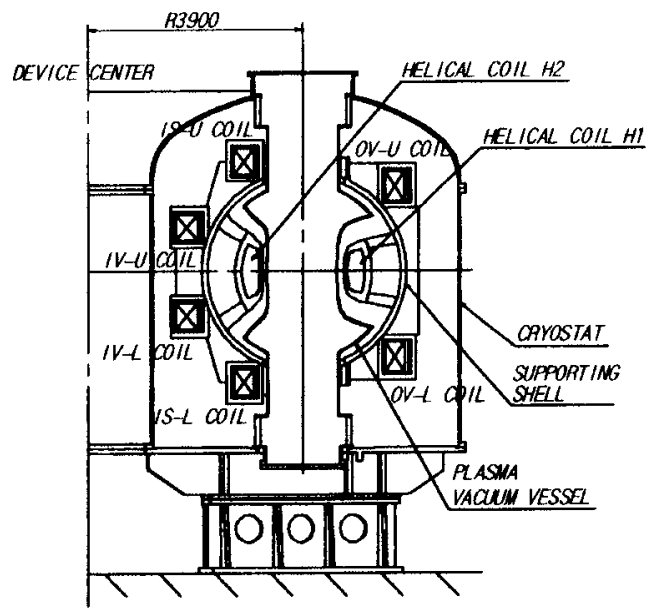

Fig. 1. Cross section of the LHD

wound in the same coil case as shown in Fig. 2.

The LHD project was planned in the following two phases. In the phase I operation, LHD operates in steady state with a magnetic field of $3 \mathrm{~T}$ at the plasma center, and in the next phase, the project is planning the experiments with a higher magnetic field of $4 \mathrm{~T}$ at the plasma center and dynamic swing of the coil currents. The current power system is designed and constructed based on the phase I operation.

The twelve coils of LHD form three poloidal coil pairs and three helical coil pairs designated as OV, IS, IV, HO, HM and HI coils respectively.

Usually, the coil power supplies start magnetization of superconducting coils in the morning, and they stop in the evening. Therefore, the coil currents flow through the day, 


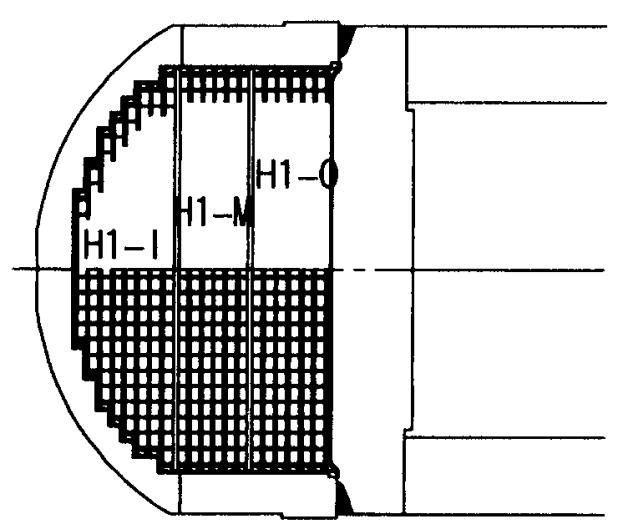

Fig. 2. Cross section of a helical coil

and the experimental group changes the experimental condition without stopping excitation ${ }^{(4)}$.

For this operation, the plasma experimental division presents the following requirements.

(1) Magnetic field error meaning the deviation from the designed value should be less than $10^{-4}$ to control the plasma position in a few millimeters.

(2) The ramp-up time from zero to the rated current and fall time from the rating to zero are less than $40 \mathrm{~min}$ utes.

(3) Magnetic field configuration can be changed within 5 minutes.

These requirements are related to the accuracy of current control and the output voltage.

\section{Outline of Power Supplies of LHD}

3.1 Main Circuits of Power Supplies Figure 3 shows the main circuits of the dc power supplies for steady state excitation for $\mathrm{HO}$ and OV coil sets. Similar power supplies are constructed for other coil sets. Table 1 shows the specifications of the power supplies. In this table, the output currents for poloidal coils were set to those of phase I operation to reduce the initial cost of the power supplies.

The output voltages of power supplies are only $33 \mathrm{~V}$ or $45 \mathrm{~V}$ in spite of the large operating current of from $16 \mathrm{kA}$ to $24 \mathrm{kA}$. In a low voltage system like this, the voltage drop in the circuit, especially forward drop in the thyristors, should be reduced as possible. So we use a 12-phase double star connected thyristor rectifier for each power supply as shown in Fig. 3.

In the LHD plasma experiments, the coil currents flow unidirectionally except the IS coil, so the power supplies are based on a single rectifier. For the IS coil, the current reverses in some magnetic confinement and mode changes related to these confinements occur infrequently. In some plasma operations, the reverse currents are required. Therefore, we decided to use a mechanical cross switches in the power supply to reverse the coil currents.

For the LHD superconducting coils, the coil diagnostic device requires sufficiently small ripple for terminal voltage to make a high quality quench detection signal. To satisfy this requirement, the power supply includes a passive dc filter at the DC output of the rectifier. Although this filter may obstruct a quick response of current control, it is not a severe

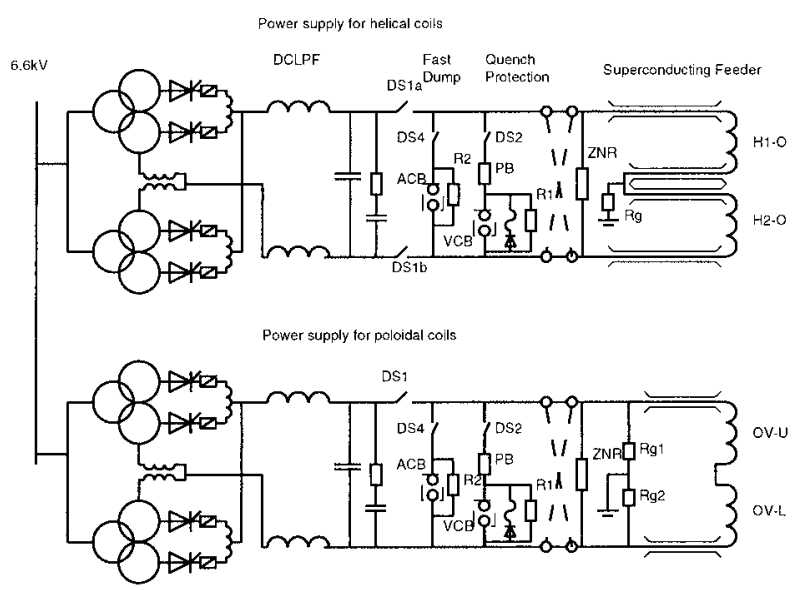

Fig. 3. Diagram of the power supplies for LHD

Table 1. Rating of power supplies for stead state operation

\begin{tabular}{ccccc}
\hline & HO, HM, HI & OV & IS & IV \\
\hline Voltage & $45 \mathrm{~V}$ & $33 \mathrm{~V}$ & $33 \mathrm{~V}$ & $33 \mathrm{~V}$ \\
Current & $17.3 \mathrm{kA}$ & $23.5 \mathrm{kA}$ & $16.3 \mathrm{kA}$ & $15.7 \mathrm{kA}$ \\
\hline
\end{tabular}

problem. Because the LHD operates in the steady state now and the current change is sufficiently slower than the filter response. We select the cut-off frequency as $50 \mathrm{~Hz}$, that is about one tenth of the ripple frequency of $720 \mathrm{~Hz}$ and it is sufficiently high compared with the current change rate.

The resonance frequency of the filter capacitor and the load coil is on the order of $1 \mathrm{~Hz}$ and is much lower than rectifier control response frequency. Therefore, a current regulator introduced in the following section can suppress this resonance.

\section{Control System for Power Supplies}

Figure 4 shows the structure of a computer subsystem for the steady state power supplies. Each power supply has a current sensor and a local feedback loop and it controls the output voltage to track the voltage reference generated by the current controller. This local controller compensates the error such as a voltage drop in the circuit or a change in line voltage then the power supply works as an ideal controlled voltage source. One real time computer controls 3 power supplies for helical coils and another one controls 3 power supplies for poloidal coils. A supervisor workstation serves user interface and development environment. A remote terminal set in the LHD main control room also can operate the control system and usually an operator commands via this terminal. This computer block is linked with LHD control system using Ethernet and a real time plasma control computer with a high-speed optical link. With these computers, the operating coil current can be set automatically.

\section{Current Controller}

In this section, we describe the current control. The performance required to the regulator is show in Table 2. The required accuracy shown in this table means that the steady state control error must be less than $0.01 \%$ of set value $+1.5 \mathrm{~A}$.

A control program controlling all power supplies, is installed in a computer system that connected to the power supplies with communication lines. This program keeps 8 


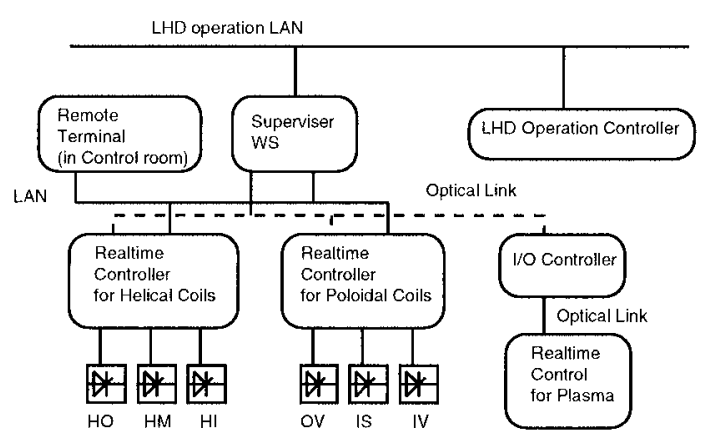

Fig. 4. Block diagram of the computer system of LHD power system

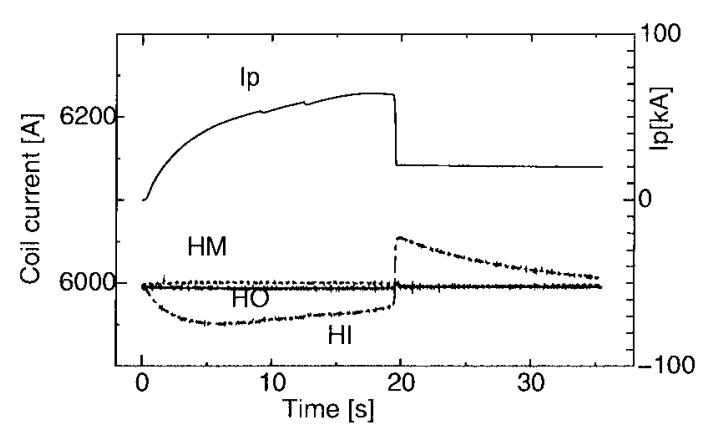

Fig. 5. Reaction caused by plasma current

Table 2. Requirements for current control system

\begin{tabular}{cr}
\hline Current measurement error & less than $(0.03 \%$ of reading value $+4.5 \mathrm{~A})$ \\
Steady state current control error & less than $(0.04 \%$ of set value $+6 \mathrm{~A})$ \\
not allowed \\
$\begin{array}{c}\text { Surrent overshoot at normal ramp up rate } \\
\text { Settling time for } 0.1 \%\end{array}$ & less than $1 \mathrm{~s}$ for normal ramp-up rate \\
\hline
\end{tabular}

Table 3. Estimated parameters for superconducting coil system of LHD

\begin{tabular}{c|cccccccccc}
\multicolumn{10}{c}{ (a) Inductance matrix [H] } \\
\hline & HO & HM & HI & OV & IS & IV & C1 & C2 & C3 & C4 \\
\hline HO & 1.343 & 1.206 & 1.052 & 0.466 & 0.355 & 0.237 & 0.874 & 0.648 & 0.706 & -0.24 \\
HM & 1.206 & 1.279 & 1.156 & 0.464 & 0.335 & 0.238 & 0.858 & 0.646 & 0.692 & -0.24 \\
HI & 1.049 & 1.154 & 1.260 & 0.462 & 0.325 & 0.238 & 0.767 & 0.611 & 0.657 & -0.21 \\
OV & 0.458 & 0.456 & 0.455 & 1.258 & 0.285 & 0.144 & 0.670 & 0.840 & -0.140 & 0.485 \\
IS & 0.329 & 0.329 & 0.329 & 0.284 & 0.953 & 0.251 & 0.405 & 0.550 & 0 & -0.15 \\
IV & 0.233 & 0.233 & 0.234 & 0.142 & 0.250 & 0.744 & 0.278 & 0.453 & 0 & -0.15 \\
C1 & 0.875 & 0.858 & 0.767 & 0 & 0 & 0 & 1 & 0 & 0 & 0 \\
C2 & 0 & 0 & 0 & 0.840 & 0.550 & 0.430 & 0 & 1 & 0 & 0 \\
C3 & 0.706 & 0.692 & 0.657 & 0 & 0 & 0 & 0 & 0 & 1 & 0 \\
C4 & 0 & 0 & 0 & 0.485 & 0 & 0 & 0 & 0 & 0 & 1 \\
\hline
\end{tabular}

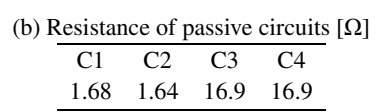

different current controllers and each controller is described with state vector as following;

$$
\begin{aligned}
& s X_{c}=A_{c} \cdot X_{c}+B_{c} \cdot\left[\begin{array}{c}
I_{c}^{*} \\
I_{c}
\end{array}\right] \\
& V_{c}^{*}=C_{c} \cdot X_{c}+D_{c} \cdot\left[\begin{array}{c}
I_{c}^{*} \\
I_{c} \\
s I_{p}
\end{array}\right]
\end{aligned}
$$

where $X_{c}$ is a state vector, $A_{c}, B_{c}, C_{c}$ and $D_{c}$ are the matrix of the control gains. $I_{c}^{*}, I_{c}, I_{p}$ and $V_{c}^{*}$ denote the current reference, the actual coil current, plasma current and the output voltage reference, respectively.

\section{Parameter Estimation of Coil System}

In the design of a control system, the electrical parameters of the superconducting coils are necessary to determine the control gains mentioned above. The inductance matrix of superconducting coils and structures was measured from the coil terminals ${ }^{(6)}$, and they are obtained as Table 3.

In the table, from $\mathrm{C} 1$ to $\mathrm{C} 4$ are equivalent circuits, which show coupled structures. This inductance matrix is asymmetric and some terms are negative. If a current flowing $\mathrm{C} 1$, $\mathrm{C} 2, \mathrm{C} 3$ and $\mathrm{C} 4$ are real current the matrix should be symmetric, but they are imaginary coils and the asymmetric matrix makes no problem to design a current controller.

Essentially helical type fusion plasma does not require plasma current but some operations with plasma current are useful to study plasma dynamics. With this reason, some plasma experiments in LHD make plasma current. For these experiments and some coil current controllers requires mutual inductance between coils and plasma. But the plasma has a large cross section and current density in the plasma is difficult to calculate. Therefore, we estimate the mutual inductance using experimental results.

Figure 5 shows the induction caused by the plasma current when the $P$ controller with $k=0.1$, which will be mentioned in the following section, is used. In this figure, the curve of plasma current does not return to zero after a plasma operation because the signal of $d i_{p} / d t$ becomes too large and it exceeds the input signal range of the control system.

In this shot, the plasma current rises to $55 \mathrm{kA}$ in 10 seconds and shuts down suddenly. When the plasma current disrupts, its induction increases the HI coil current.

Figure 6 shows plots of the coil current deviation when plasma experiments with plasma current were done. The horizontal axis is for the HI coil and the vertical axis is for the IV coil. The point of $I_{H I}=11.0 \mathrm{kA}$ and $I_{I V}=11.2 \mathrm{kA}$ shows a steady state operation point. The plots exist surround a line with slope of 0.324 as shown in the figure. This means that the ratio of mutual coupling between plasma, HI and IV coils is constant, and the plasma can be described with a one turn coil for the control system design.

The mutual inductances between plasma and coils are estimated by the following way.

When plasma current disrupts, the interlinked fluxes to the 


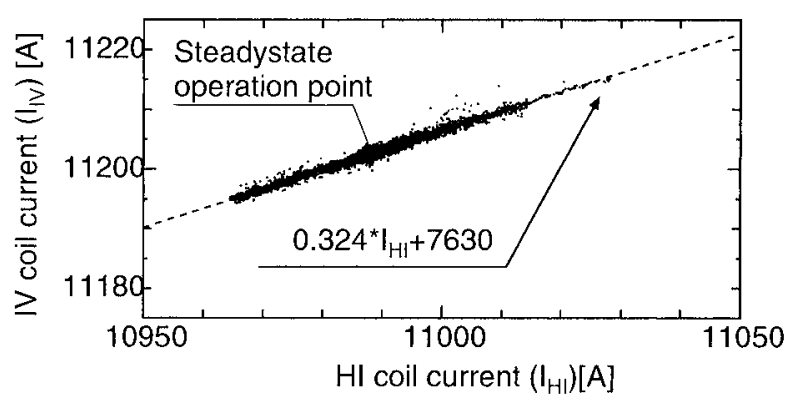

Fig. 6. Plots of the coil current deviation

Table 4. Mutual inductance between coils and plasma [unit $\mathrm{mH}$ ]

\begin{tabular}{cccccc}
\hline HO & HM & HI & OV & IS & IV \\
1.60 & 1.69 & 1.80 & 1.05 & 0.88 & 0.67 \\
\hline
\end{tabular}

coils are almost constant because the time constant of the coil current control is enough longer. Therefore circuit equation in this situation is described as follows;

$$
\Delta \psi=L_{c} \Delta i_{c}+M_{c p} \Delta i_{p}=0
$$

where $L_{c}, M_{c p}, \Delta i_{c}, \Delta i_{p}$ and $\Delta \psi$ are self-inductance of coils, mutual inductance between coils and plasma, coil current deviation, plasma current deviation and change of interlinked flux respectively. From this equation, $M_{p c}$ can be calculated as following;

$$
M_{p c}=-L_{c} \Delta i_{c} / \Delta i_{p}
$$

Table 4 shows the estimated mutual inductances and they are about two third of calculated value which are obtained using a model that assumes a line current at the plasma center.

\section{Basic Current Control Schemes}

In this section, the basic current control schemes for LHD power system are described.

7.1 PI Controller First, the PI control scheme, which does not use a signal of plasma current, is designed for this system. Although this control scheme is simple, it has adequate performance for the steady state current control.

The current regulator used in this system is formed as follows;

$$
V_{c}^{*}=\frac{k}{s} R_{c}\left(I_{c}^{*}-I_{c}\right)+k L_{c}\left(I_{c}^{*}-I_{c}\right)
$$

where $L_{c}$ is inductance matrix of the superconducting coils, $R_{c}$ is resistance, and $\mathrm{k}$ is the scalar control gain. From the equations, the control matrix in equation 1 and 2 are evaluated as follows;

$$
\begin{aligned}
& A_{c}=0, B_{c}=\left[\begin{array}{cc}
U & 0 \\
0 & -U
\end{array}\right], \\
& C_{c}=k R_{c}, D_{c}=k\left[\begin{array}{cc}
L_{c} & 0 \\
0 & -L_{c}
\end{array}\right]
\end{aligned}
$$

where $U$ is a unit matrix. The step response of the coil current is as following;

$$
I_{c}=I_{c 0}+\frac{1}{1+s \tau}\left(I_{c}^{*}-I_{c 0}\right)
$$

where $\tau=1 / k$ is a characteristic time constant, $I_{c 0}$ is an initial value of $I_{c}$. From this equation, it is clear that the every coil current has the same response and the balance of the coil currents are kept during the transient.

The control gain $k$ deciding the system response has upper limit decided by the stability. This limit is determined due to the control theory as $k<2 \pi f_{c} / 4$, where $f_{c}$ is a cut-off frequency of a low pass filter inserted to reduce noise. In the LHD power system, the $f_{c}$ is selected as $0.87 \mathrm{~Hz}$, therefore the upper limit of $k$ becomes 1.3.

The $P$ control scheme is obtained by setting of $R_{c}=0$ in the equation (5). In this $P$ control, the steady state control error $I_{e}=I_{c}^{*}-I_{c}$ remains as following;

$$
I_{e}=\left(k L_{c}\right)^{-1} \cdot R_{c} \cdot I_{c}^{*} \text {. }
$$

In this system, $I_{e}$ may become about $0.01 \%$ of $I_{c}^{*}$ when $k=1.0$ because the orders of $R_{c}$ and $L_{c}$ are $10^{-4}$ and 1 , respectively.

Table 5 shows the system size and some characteristic parameters for each control system. The $H \infty$ control and advanced control schemes will be described later. When we use the $P$ control, $k=1$ is necessary to satisfy the requirements for control error and response time as shown in the table. Figure 7 shows a ramp response of the $P$ controller with $k=1$. In this figure, the $\mathrm{HO}$ current reference is swept and the other current references are kept constant. The HO current traces its reference with $1 \mathrm{~s}$ delay and the disturbance of the other currents are less than $2 \mathrm{~A}$. With this result, the independent current control for each coils are confirmed. For the $P I$ controller, a similar result is observed.

In the other viewpoint, the eddy current flowing in the structures changes when the coil current reaches to stead state value. This eddy current induces a noise signal to quench detector, and it becomes larger if the corner of the coil current waveform is too sharp. The low gain $P$ controller makes soft landing of coil current to the steady state, and they causes only low induced voltage to the quench detector. Also the low gain $P$ control scheme has following advantages; it is very stable, terminal voltage ripple is small and the induced noise to quench detector is also small. With these reason, the $P$ controller with $k=0.1$ is used in usual plasma experiments even though it does not satisfy the specification of accuracy and settling time.

7.2 Hळ Controller In the PI controller mentioned above, the control gain has limitation because of the stability requirement. The $H \infty$ using $\mu$-synthesis design scheme is one of the solutions to manage both the stability and fast response ${ }^{(5)}$. The $H \infty$ controller using $\mu$-synthesis design method has robustness for control performance as well as stability and uncertainty of the controlled subject can be taken into consideration.

In Fig. 8, $P$ shows the controlled object, i.e., LHD coils, $K$ shows the controller obtained by the $H \infty$ theory, and $G_{m}$ shows a transfer function to determine the output response of coil current to the current reference. The $G_{m}$ should be specified properly to get the desired output characteristics. In this controller, the feed forward controller calculates the coil voltage required to follow the current reference. The response of coil current to various disturbances can be determined by the feedback controller $K$. This control scheme can determine 
Table 5. Comparison of the current control system

\begin{tabular}{c|cccc|ccc}
\hline & \multicolumn{4}{|c|}{ Basic control scheme } & \multicolumn{3}{c}{ Advanced control scheme } \\
\hline Control scheme & $\mathrm{P}$ & $\mathrm{P}$ & $\mathrm{PI}$ & $H \infty$ & $H \infty(1)$ & $H \infty(2)$ & $\begin{array}{c}\text { Advanced P control } \\
\end{array}$ \\
& $k=0.1$ & $k=1$ & $k=1$ & & & & $k=0.1$ \\
\hline Size of $X_{c}$ & 6 & 6 & 12 & 56 & 62 & 68 & 18 \\
Numbers of parameters & 84 & 84 & 108 & 4,200 & 5,028 & 5984 & 186 \\
Steady state control error & $0.1 \%$ & $0.01 \%$ & $<0.01 \%$ & $<0.01 \%$ & $<0.01 \%$ & $-(* 1)$ & $<0.01 \%$ \\
Response time constant $(* 2)$ & $10 \mathrm{~s}$ & $1 \mathrm{~s}$ & $1 \mathrm{~s}$ & $<0.3 \mathrm{~s}$ & $<0.3 \mathrm{~s}$ & $<0.3 \mathrm{~s}$ & $1 \mathrm{~s}$ \\
\hline
\end{tabular}

$(* 1)$ There is a current drift and steady state error cannot be defined.

(*2) For the major components. There are longer time constant components in some scheme but their amplitude is small enough.

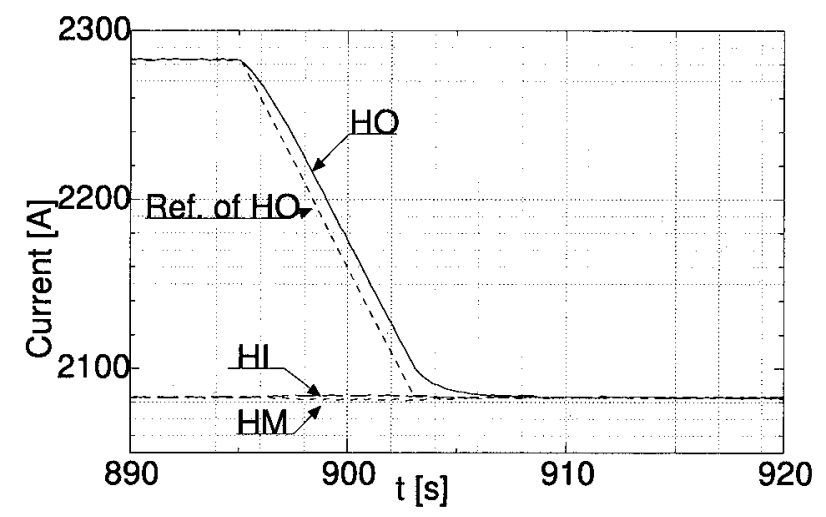

Fig. 7. Ramp response of the coil current with $P$ control $(k=1)$

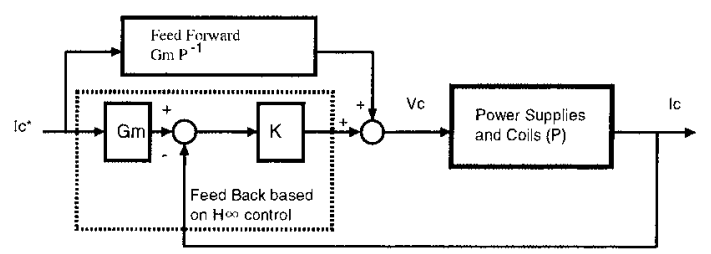

Fig. 8. Block diagram of $H \infty$ control for LHD power supply

response characteristics to disturbances and current reference independently, so it is called as two-degree-of-freedom control scheme. The function of $G_{m}(s)$ was chosen $g_{m}(s) U$. Then $G_{m}(s)$ becomes a diagonal matrix and the interaction of each coil current can be avoided by the zero component of this matrix. The $g_{m}(s)$ was chosen as $g_{m}=\{8 /(1+8 s)\}^{2}$ from the simulation results of the closed loop controller to get the fastest response without overshoot for stepwise current reference.

The $H \infty$ controller was designed as follows. A block diagram of the design problem is shown in Fig. $9 . P_{n}$ shows the nominal model of the plant, $W_{a}$ shows the frequency weighting function for modeling error, $W_{M}$ shows the frequency weighting function for disturbance, $\Delta$ shows the additive modeling error, $\Delta_{F}$ shows the fictitious uncertainty which introduces disturbance. $W_{a}$ and $W_{M}$ are diagonal matrix and in the relation of trade off each other. We made some simulations and set the weighting functions as shown in Fig. 10 for this power supply. The gain and phase characteristics of the $H \infty$ controller is shown in Fig. 11.

Figure 12 shows a ramp response of the $H \infty$ controller designed for LHD and it is clear that the $H \infty$ control realizes the better response than the $P I$ controller.

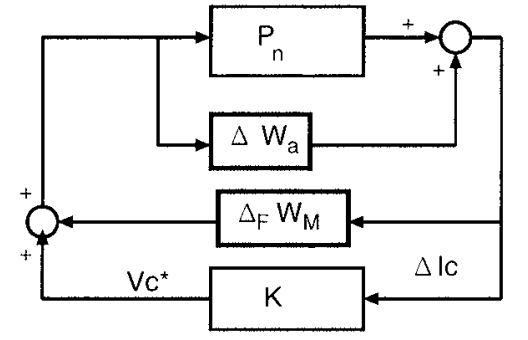

Fig. 9. Block diagram for $H \infty$ control design

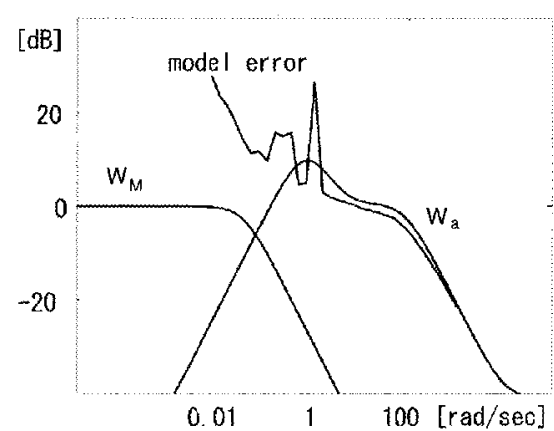

Fig. 10. Gain characteristics of weighting function $W_{M}$ and $W_{a}$

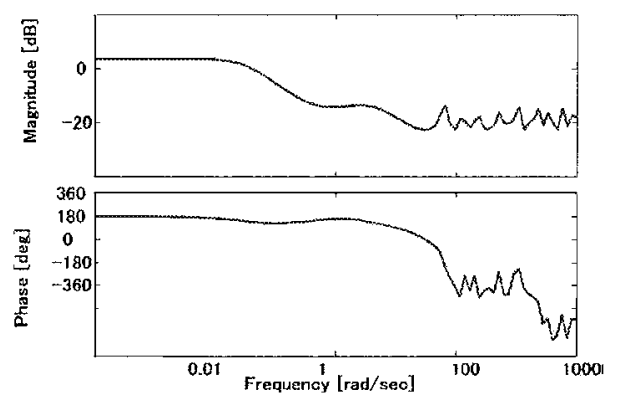

Fig. 11. Gain and phase characteristics of the $H \infty$ controller

\section{Advanced Current Control Schemes}

In the previous section, basic $P I$ and $H \infty$ control schemes are described. These control schemes are stable enough but could not suppress the coil current deviation caused by the plasma current even if the $H \infty$ control was applied. To resolve the problem, we designed advanced controllers based on the $H \infty$ control.

On the other hand, the $P$ control with low control gain $K$ is also useful mentioned above. A current error observer and feed forward control are useful to improve the current 


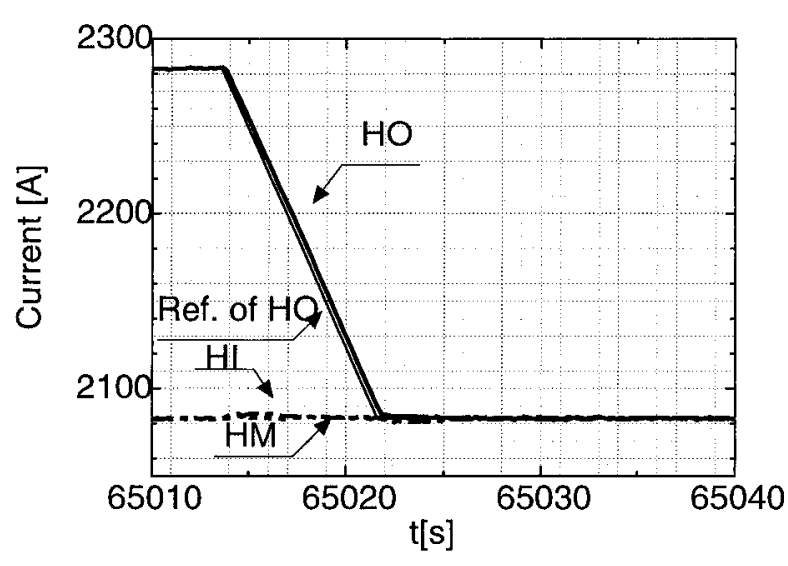

Fig. 12. Ramp response of the coil current with $H \infty$ control

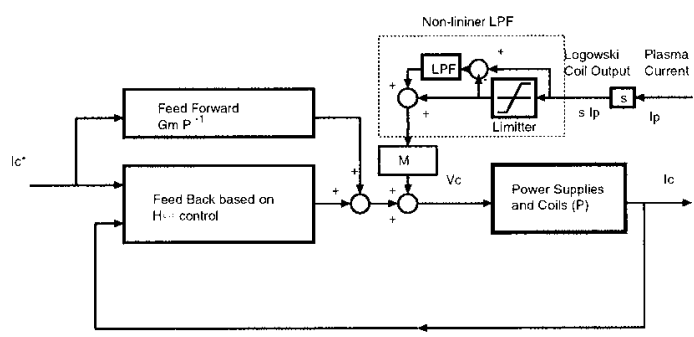

Fig. 13. Block diagram of $H \infty$ (1) controller

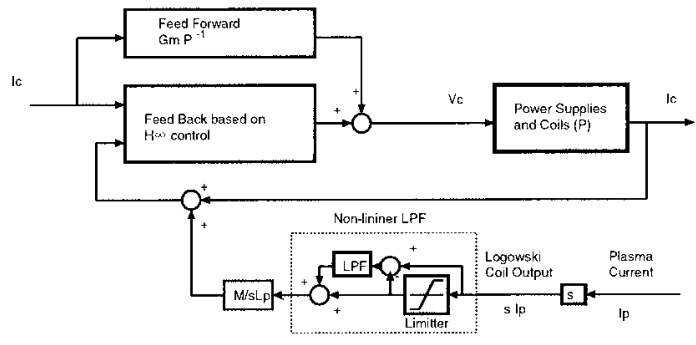

Fig. 14. Block diagram of $H \infty$ (2) controller

response and steady state error. Then we also designed one advanced controller based on the $P$ control.

8.1 Advanced $H_{\infty}$ Controllers The information of the plasma current is useful to reduce the interaction to the coil currents from the plasma current. Then we designed two types of advance $H \infty$ controllers, which use the plasma current signal and have different characteristics, for the LHD power system.

One, named $H \infty(1)$, is designed to keep the coil currents constant even if the plasma current is excited. The block diagram is shown in Fig. 13. This controller uses the plasma current detected by Logowski coil to generate feed forward term for the coil voltage. A non-linear LPF inserted to limit the level of Logowski coil output as shown in the figure. The other one, named $H \infty(2)$, is designed to keep magnetic flux constant while a plasma experiment. This controller estimates the coil current deviation induced by change of plasma current and allows the deviation. Figure 14 shows a control diagram of $H \infty$ (2) controller. With the $H \infty$ (2) control, the coil currents may be changed when plasma current is excited but the terminal voltages will be kept as almost zero.

Figure 15 show the waveforms of coil currents (HO, HM,
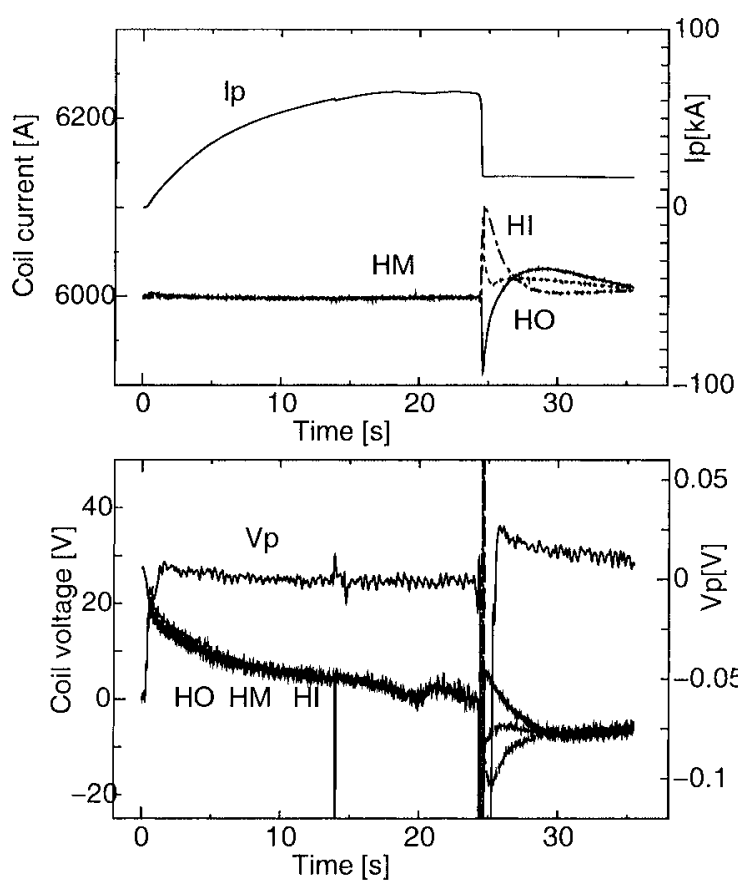

Fig. 15. Voltage and current waveforms with $H \infty(1)$ control scheme
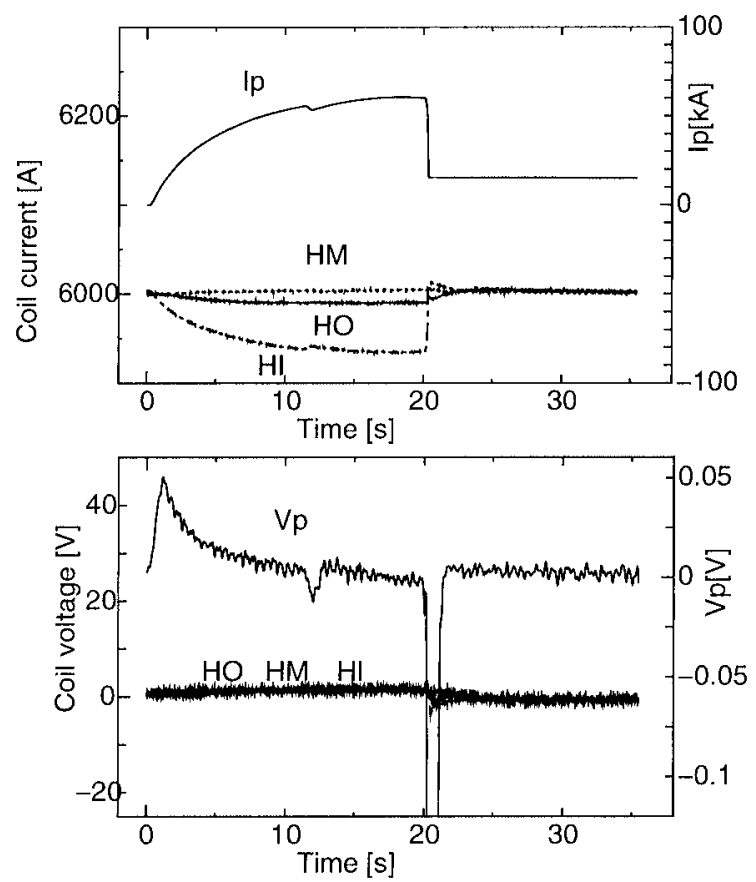

Fig. 16. Voltage and current waveforms with $H \infty(2)$ control scheme

$\mathrm{HI}$ ), plasma current $I_{p}$, coil terminal voltages (HO, HM,HI) and calculated plasma induced voltage $V_{p}$ using the $H \infty(1)$ control. Also Fig. 16 shows the waveforms using the $H \infty$ (2) control. In these experiments, we wanted to show the effect to the plasma current caused by coil current controllers and the non-linear LPF was not used to make simple the response. Figure 15 shows that the controller kept coil currents constant while plasma current ramp up and it reached flattop. When the plasma breaks, the $d I p / d t$ signal becomes too large and the calculated output voltage exceeded the maximum 


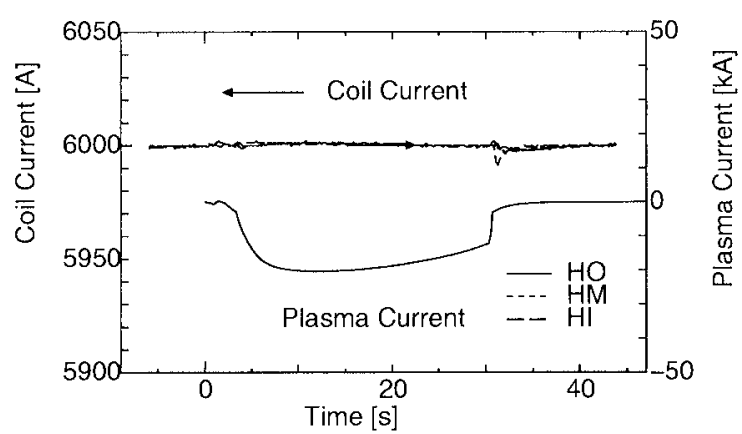

Fig. 17. Current waveforms with $H \infty(1)$ control scheme

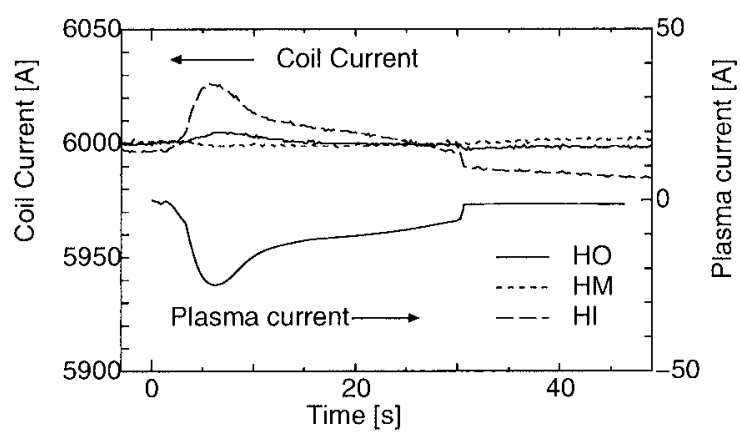

Fig. 18. Current waveforms with $H \infty(2)$ control scheme

output voltage of the power supplies. With this reason the controller was disturbed and the coil currents show some transient waveforms but it did not cause any instability. In Fig. 16, the controller keeps the interlinked magnetic flux and the coil current show some deviations. As shown in the figure, the coil terminal voltages were almost zero because the interlink flux were not changed. When this scheme was applied to the LHD, it is clear that the rebound of coil current when the plasma shut down was smaller than the case of Fig. 15, and coil currents returned to their references immediately. With comparing these two operation, the induced voltage to a plasma when the ip ramps up and ramp up rate of $i p$ are different. When $H \infty$ (2) was used, the induced voltage becomes lager and plasma current ramp up faster. In the figures, $t=10 \mathrm{~s}$, a plasma heating device was changed. At this point, the plasma current and induced voltage changed lager when $H \infty(2)$ control was used.

Figures 17 and 18 show the current waveforms when the $H \infty$ (1) and $H \infty(2)$ control schemes with non-linear LPF were used. The non-linear LPF clipped the peak of $s I p$ signal and the calculated output voltage did not exceed the rated output voltage. Therefore the coil currents and terminal voltages were not disturbed when plasma discharge is finished. In Fig. 18, the coil current drifted while plasma discharged in the operation. A dc offset voltage of the non-linear LPF causes this drift. Even though the long-term operation using this control scheme is difficult with this drift, usual plasma experiments are shorter than $30 \mathrm{~s}$ and this scheme can be applied.

8.2 Advanced $\boldsymbol{P}$ Control The last control scheme is advanced $P$ control using additional a current error observer and feed forward shown as in Fig. 19.

Comparing this control scheme with $P I$ control or $H \infty$

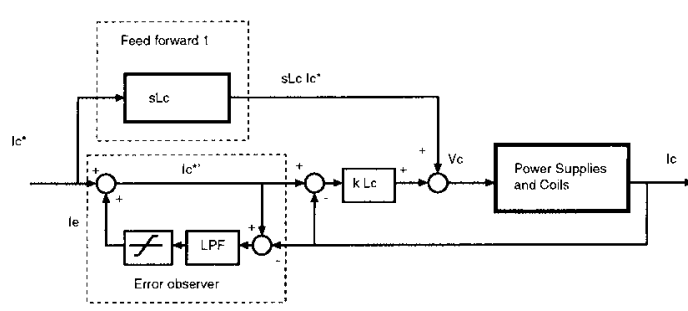

Fig. 19. Block diagram of an advanced $P$ control scheme

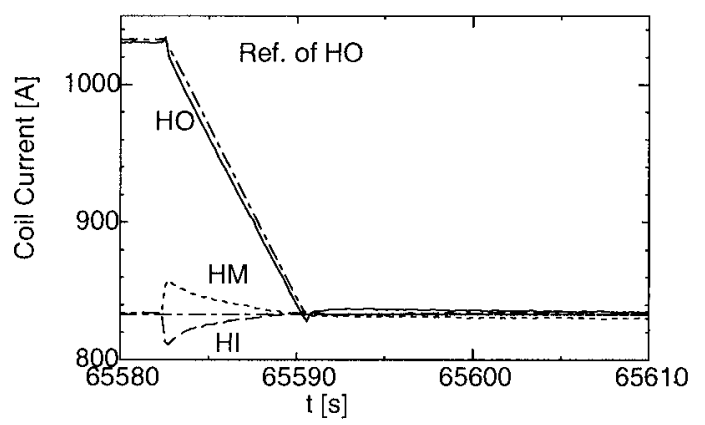

Fig. 20. Ramp response of the coil current with advanced $P$ control $(k=0.1)$

control scheme, the limiter to the observed error can be inserted at the observer output. With this limiter, the large turbulence caused by a spike noise in the current data can be avoided.

Figure 20 shows a result of current ramp test of this controller. As the result shows, this controller has satisfies the requirements for the response and the steady state error even if the control scheme is more simple than the $H \infty$ control schemes.

8.3 Dynamic Swapping of Current Controller In usual plasma operations, the $P$ control scheme with $K=0.1$ is used. For some LHD plasma experiments such as a plasma current feedback operation, this controller cannot satisfy requirements of dynamic characteristics and it is necessary to change the control scheme. Therefore we install ability of dynamic swapping of current controller in the control program.

To swap a control scheme, the program must initialize the internal variables in the controller to a suitable value to avoid the large transients

When the control system is in steady state and a current control error is small enough, $s X_{c}=0$ and $I_{c}^{*}=I_{c}$ are kept in the equation (1). Then the state variable $X_{c}$ becomes as following;

$$
X_{c}=[U-A]^{-1}\left[B_{1}+B_{2}\right] I_{c}^{*} .
$$

This $X_{c}$ is used as an initial value.

For $P I$ control scheme, matrix $\mathrm{A}$ is singular and $X_{c 0}$ cannot be calculated with above equation. In this case, the state equations are transformed into following form and initial vector $X_{c 0}$ is calculated;

$$
\left[\begin{array}{l}
X_{c} \\
X_{i}
\end{array}\right]=\left[\begin{array}{cc}
A_{1} & A_{2} \\
A_{3} & U
\end{array}\right]\left[\begin{array}{c}
X_{c} \\
X_{i}
\end{array}\right]+\left[B_{1}, B_{2}\right]\left[\begin{array}{c}
I_{c}^{*} \\
I_{c}
\end{array}\right]
$$




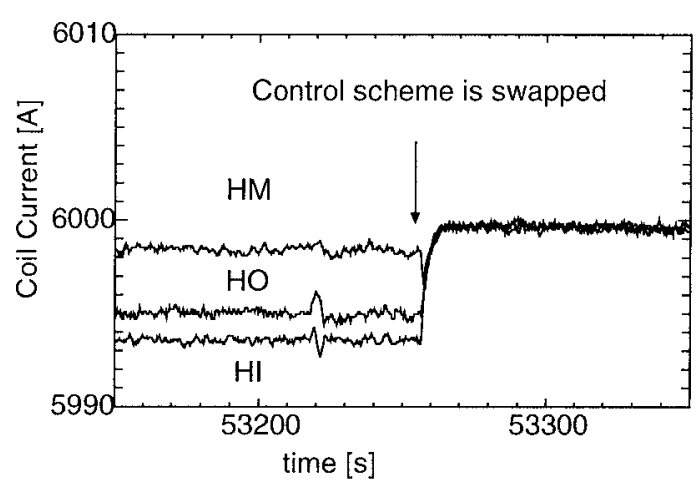

Fig. 21. Swap of control scheme from usual $P$ controller to a high gain $P$ controller

$$
\begin{gathered}
V=\left[C_{1} C_{2}\right]\left[\begin{array}{c}
X_{c} \\
X_{i}
\end{array}\right]+\left[D_{1}, D_{2}\right]\left[\begin{array}{c}
I_{c}^{*} \\
I_{c}
\end{array}\right] \ldots \ldots \ldots \ldots \\
{\left[\begin{array}{c}
X_{c 0} \\
X_{i 0}
\end{array}\right]=\left[\begin{array}{c}
{\left[U-A_{1}\right]^{-1}\left[B_{1}+B_{2}\right] I_{c}^{*}} \\
0
\end{array}\right] \ldots \ldots \ldots}
\end{gathered}
$$

Figure 21 shows the current waveforms when control scheme was swapped from a $P$ controller with $K=0.1$ to the $P$ controller with $K=1.0$. Even though the $P$ control with $K=0.1$ causes a steady state error, the control scheme can be changed without any turbulence. and it becomes an initial offset for high gain controller, each control scheme is stable enough and it did not make any problem.

With this ability of swapping the current control scheme even if the coils are magnetized, the power system becomes more useful for plasma experiments.

\section{Conclusions}

This paper describes the current control system in the LHD dc power system. To design the current controller, the electrical parameters of the coil system are estimated and several types of the controllers are designed and tested. The test results show that these controllers are stable and perform required characteristics. The control scheme can be selected with regard to the requirements from plasma experiment. The developed current control systems will contribute to the study on the plasma response characteristics under different coil current controls.

\section{Acknowledgment}

The power system of the LHD superconducting coil system is constructed under the cooperation with Aichi electric co., ltd., Kobe steel co., ltd., Densan co., ltd. and Mitsubishi space software. In addition, many staffs of the project team support LHD experiments. Moreover, this work is performed under the auspices of the NIFS Collaborative Research Program. The authors would like to thank the staffs of the LHD project and cooperated companies. This research was partially supported by the general coordinated research program of NIFS. The authors thank to the related persons. Also the parts of this study were supported by NIFS research program, which codes are NIFSULAA110 and NIFS05ULAA509.

(Manuscript received May 9, 2005, revised Dec. 13, 2005)

This paper was presented at IPEC-Niigata 2005, and approved for publication in the IEEJ Transactions on Industry Applications Society.

\section{References}

(1) A. Iiyoshi et al.: "Overview of the Large Helical Device project", Nuclear Fusion, Vol.39, spec. issue., pp.1245-1256 (1999)

( 2 ) O. Motojima et al.: "Physics and engineering design studies on the LHD", Fusion Eng. and Design, Vol.20, pp.3-14 (1993-1)

( 3 ) T. Satow: "Specifications and performance experience of pool boiling cooled magnets", Cryogenics, Vol.38, pp.1145-1153 (1998-11)

( 4 ) S. Tanahashi et al.: "Design study of power supplies for LHD superconducting magnets", Fusion Eng. and Design, Vol.20, pp.107-111 (1993-1)

( 5 ) T. Ise et al.: "Current control of magnetically coupled super conducting coils for nuclear fusion experimental system using $H \infty$ control scheme", PESC 96 Record. 27th Annual IEEE Power Electronics Specialists Conf., pp.1099_ 1104 (1996)

( 6 ) H. Chikaraishi et al.: "Estimation of electrical parameters of large scale superconducting coil system for fusion plasma experimental facility", IEEE Trans. on Applied Super., Vol.12, No.1, pp.1374-1377 (2002)

Hirotaka Chikaraishi (Member) was born in Hiroshima, Japan,

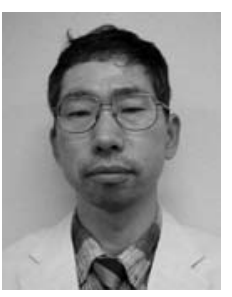
1964, and received a Ph.D. degree of Engineering from Tokyo Institute of Technology, Tokyo, Japan. He joined LHD group of National Institute for $\mathrm{Fu}-$ sion Science in 1992, and is associated professor. His research interests are power systems for the fusion power facility.

Shigeyuki Takami (Non-member) was born in Gifu, Japan, 1974, and

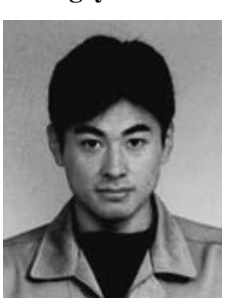
graduated from Gifu University, Gifu, Japan. He joined the NIFS in 1997, where he works on developing a control system.

Tomoyuki Inoue (Non-member) was born in 1970, and graduated

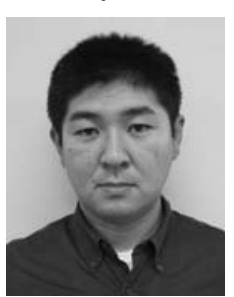
from Gifu University, Gifu, Japan. He joined LHD group of National Institute for Fusion Science in 1996.

Satoru Sakakibara (Non-member) was born in Osaka, Japan, 1966,

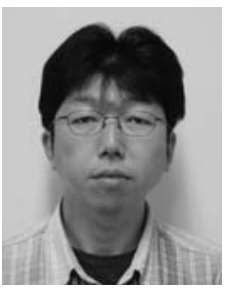
and received a Ph.D. degree of Engineering from Graduate University for Advanced Studies, Hayama, Japan. He is an associate professor at the National Institute for Fusion Science. He is responsible for magnetic measurements in Large Helical Device (LHD) and his current interests include MHD equilibrium and stability in high-beta helical plasmas. 
Toshifumi Ise (Member) was born in 1957. He received the Bachelor, Master, and Dr. of Engineering degrees from Osaka University, Osaka, Japan, in 1980, 1982 and 1986, respectively, all in electrical engineering. From 1986 to 1990, he was with the Nara National College of Technology. In July 1990, he joined the Department of Electrical Engineering, Faculty of Engineering, Osaka University, and is Professor from 2002. His research interests are in the areas of power electronics and applied superconductivity including superconducting magnetic energy storage (SMES), new distribution system for distributed generations and power quality.

Tomoyuki Haga (Non-member) received the B.ES (Engineering Sci-

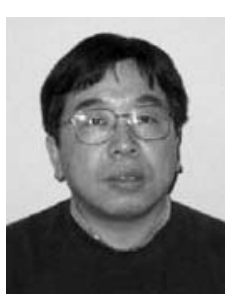
ence) in control engineering from the University of Osaka, Osaka, Japan, in 1976. He joined the Electronics Technology Institute of Kobe Steel, Ltd. and is currently with Shinko EN\&M co., Ltd. where he works on developing Image \& Control system. He is a member of the Society of Instrument and Control Engineers (SICE).
Hirohiko Niwa (Member) was born in 1956. After graduating

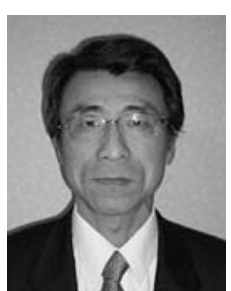
from Electrical and Electronic Engineering department in Facility of Engineering Kanazawa University in 1979, he joined Aichi Electric Co., Ltd. and engaged development of power electronics in system development headquarters. He engages the work of quality assurance and quality control in quality assurance group in Electric \& Electronic Products Division since 2003. 